\title{
Neurological disease in children linked to enterovirus D68
}

A cluster of cases of acute flaccid paralysis and cranial nerve dysfunction in children was temporally associated with an outbreak of enterovirus D68, according to researchers at Children's Hospital Colorado, USA. The link could mean that the virus is a neuropathogen, highlighting an important need for further research.

Although acute flaccid paralysis has been associated with flaviviruses and enterovirus A71, less evidence exists for a link with enterovirus D68. "Enterovirus D68 has only been associated with CNS disease in two previously published cases," explains lead researcher Kevin Messacar.

Suspicions were aroused during a 2014 outbreak of enterovirus D68 in the USA. "From August to October 2014, clinicians at Children's Hospital Colorado noted an unusual number of children presenting with distinctive symptoms of muscle weakness and distinctive neuroimaging findings," says Messacar.

Working with the Colorado Department of Public Health and Centers for Disease Control and Prevention (CDC), the
4. ...cases tripled during the enterovirus D68 outbreak relative to any 3-month period in the preceding 3 years... 77

researchers developed a case definition for the unusual neurological condition, and analysed its prevalence and association with infection.

In the 3-month study period, 12 children met the case definition, and presented with acute flaccid paralysis with spinal cord grey matter lesions and/or cranial nerve dysfunction with brainstem lesions. A febrile illness preceded neurological symptoms in all 12 children.

Messacar's team identified cases that met the definition from a historical control period, and found that the number of cases tripled during the enterovirus D68 outbreak relative to any 3-month period in the preceding 3 years: a statistically significant increase.

The researchers also tested the children for enterovirus and rhinovirus infection.
Of seven serum and 11 cerebrospinal fluid specimens collected, all tested negative, but eight of 11 nasopharynx specimens were positive, five for enterovirus D68.

"This study strengthens the potential link between enterovirus D68 and neurological disease in children," says Messacar. He remains cautious, however: "given that we did not detect the virus in spinal fluid, efforts are needed to further investigate this association."

Working closely with the CDC, Messacar and colleagues are currently devising case-control studies to further explore the association. As Messacar points out, reporting to the CDC of cases that meet the accepted definition will be essential for future research.

Ian Fyfe

Original article Messacar, K. et al. A cluster of acute
flaccid paralysis and cranial nerve dysfunction
temporally associated with an outbreak of enterovirus
D68 in children in Colorado, USA. Lancet doi:10.1016/
S0140-6736(14)62457-0

Original article Messacar, K. et al. A cluster of acute ccid paralysis and cranial nerve dysfunction D68 in children in Colorado, USA. Lancet doi:10.1016/ S0140-6736(14)62457-0 